\title{
Who is Missing in Planetary Science?: Strategic Recommendations to Improve the Diversity of the Field
}

\author{
Authors \\ Julie A. Rathbun, Planetary Science Institute, Rathbun@psi.edu \\ Edgard G. Rivera-Valentín (Lunar and Planetary Institute, Universities Space Research \\ Association) \\ James Tuttle Keane (Jet Propulsion Laboratory, California Institute of Technology) \\ Kennda Lynch (Lunar and Planetary Institute, Universities Space Research Association) \\ Serina Diniega (Jet Propulsion Laboratory, California Institute of Technology) \\ Lynnae C. Quick (NASA Goddard Space Flight Center) \\ Christina Richey (Jet Propulsion Laboratory, California Institute of Technology) \\ Janet Vertesi (Princeton University) \\ Orenthal J. Tucker (NASA Goddard Space Flight Center) \\ Shawn M. Brooks (Jet Propulsion Laboratory, California Institute of Technology)
}

\section{Co-Signers}

This white paper is submitted as part of a collaborative effort organized by the Equity, Diversity, and Inclusion Working Group (EDIWG), a cross Assessment Group (AG) committee.

As of September 15th, this paper has been co-signed by over 100 people, which can be found at https://docs.google.com/spreadsheets/d/1kUSOTvfRQ0x0Ky609Zm2p YWDDyqyFou4XgJKpLUP Rc/edit?usp=sharing.

We are still accepting new cosigners. If interested, please fill out this form: https://docs.google.com/forms/d/e/1FAlpQLSdmxZw_AEGdJY hR8UWBPKA3swhELbQcORVUq vr2GYi47Pymw/viewform?usp=sf_link

Companion paper (Paper 1; Rivera-Valentín et al. 2020):

https://drive.google.com/file/d/1n4UUIDV426ZwwTQ-0RfI5BtPOF40H5XM/view?usp=sharin g 


\section{Introduction}

Racial and ethnic minorities (particularly those of African American, Latinx, and Native American background) are significantly underrepresented in planetary science. Comparisons of the numbers of planetary scientists to the US population show that these communities are more underrepresented than white or Asian scientists by a factor of $\sim 10$. Here, we summarize the relevant demographic data available for planetary science and related fields (see companion white paper by Rivera-Valentín et al. for a detailed discussion) and share social science results that could help explain the disparity. Finally, we provide actionable recommendations that can be implemented to improve the climate for members of underrepresented groups in planetary science.

\section{Demographic results}

Rathbun, et al. (2015, 2016, 2018) has shown, using the 2011 Planetary workforce survey (White, et al., 2011), that the planetary science workforce is not nearly as diverse as the US population from which it was drawn and from which the majority of funding originates. They showed that only $25 \%$ of planetary scientists were women; while by ethnicity $87 \%$ were white, $7 \%$ were Asian, and 1\% each were Black or Latinx. The US population in 2010 was 51\% women and $64 \%$ white, $13 \%$ Black, $16 \%$ Latinx, and 5\% Asian. Hence the planetary science workforce had an overrepresentation of men relative to the general population, particularly white and Asian men. Rathbun et al. (2017) looked at the net planetary science pipeline. Their calculations demonstrated that women of color (not including Asian women) are the most underrepresented group in planetary science. Furthermore, white women are closer in representation levels to white men than to women of color: For every 3 white men that make it through the pipeline, there is 1 white woman. But, for every 20 white women, there are only 1-2 women of color. This means that more than $95 \%$ of potentially talented women of color are being left behind and thus are unable to contribute to the planetary science community.

Rivera-Valentín et al. (2020) used the 2020 Planetary workforce survey (Hendrix, et al., 2020) to show that racial and ethnic diversity in planetary science has not improved much since the 2011 survey was conducted. They also improved on the analysis of Rathbun et al. (2015, $2016,2017,2018$ ) by comparing the demographics of planetary scientists to that in the National Civilian Labor Force (NCLF). Rivera-Valentín, et al. (2020) found that representation of non-student women researchers in planetary science grew from $25 \% \pm 1.7 \%$ in 2011 to $34.7 \% \pm$ $2.5 \%$ in 2020 (i.e., a $9.7 \% \pm 3.0 \%$ increase), while at present women comprise $50.3 \% \pm 4.2 \%$ of student researchers in planetary science. Rivera-Valentín et al. (2020) also found that the representation of Latinx non-student researchers increased from $1 \% \pm 0.3 \%$ to $4.0 \% \pm 1 \%$ (i.e., an increase of $3.0 \% \pm 1.0 \%$ ), which is comparable to the national growth of the Latinx community during the same time period. However, and most egregiously, they found that no statistically significant change in the representation for Black non-student planetary scientists has occurred over the past decade. In fact, when analyzing the demographics of the current student researchers in planetary science, Rivera-Valentín et al. found that, although there is improvement for the representation of women and Native Americans, there is little to no difference between the low representation of Latinx and Black researchers between the two groups. However, this is not surprising, given that Bernard and Cooperdock (2018) found that 
there had been no increase in the number of PhDs awarded to African American geoscientists in the past 40 years.

\section{Discussion/interpretation}

\subsection{Why is Diversity important?}

Diverse workforces are proven to generate more innovative and interdisciplinary thinking. Groups where individuals come together from different backgrounds offer new solutions to intractable problems, leading to both technological and scientific breakthroughs (Philips, 2014). By contrast, places with high levels of homogeneity in their workforce are subject to groupthink and risk (Neff, 2012; Vaughan, 2016). In particular, people from underrepresented minority groups produce higher rates of scientific innovation (AlShebli et al., 2018), but their work is systematically undervalued (Hofstra et al., 2020).

Rathbun et al., 2016 pointed out that the 2011 planetary science workforce survey showed that $72 \%$ of planetary science research is supported by US public research funds (NASA and NSF). Since funds are from the public, ensuring public support of our scientific endeavors is particularly important for planetary science. As our workforce threatens to become increasingly less diverse than the US population, it will become difficult for much of the US public to see themselves engaged in planetary science, and public support will likely wane.

Underrepresentation of Latinx and African American planetary scientists is NOT due to differences in interest or ability. O'Brien et al. (2015) found that African American first year college students indicate an interest in majoring in science at higher rates than their white peers ( $23 \%$ vs. $16 \%)$. There are also no measurable differences in entrance in STEM majors among White, African American, and Hispanic ${ }^{1}$ students (Chen \& Weko 2009 ).

\subsection{Isn't planetary science a meritocracy?}

In industries that pride themselves on meritocratic advancement, such as planetary science, one might suggest that the best junior participants will rise to the top. This belief has been disproved by a barrage of sociological studies of the sciences and technical domains. For example, studies have shown that beliefs in "innate talent," meritocracy, and colorblind approaches can yield more unequal access and participation. In this section, we list specific ways these colorblind approaches fail, along with references.

Being reminded of minority status negatively affects people's performance (Steele and Aronson, 1995). Also, minorities who attempt to take leadership roles acquire negative reputations because they are perceived as deviant (Rudman and Glick, 2001). Experimental studies that change the name on a resume have shown the tremendous effects of implicit bias at play in evaluation of female and racial minority candidates for promotion, support, or hiring (e.g. Foschi, et al., 1994), more so during economic hard times (Thébaud and Sharkey, 2016). Meanwhile, women who are judged "competent" are typically held back in their careers instead of offered opportunities to advance (Ridgeway, 2011) or hold themselves back so as to remain in-line with gendered expectations (Correll, 2004).

Masculine work cultures can create self-fulfilling prophecies, where the right person for the technical or scientific job can only be white and male (Ensmenger, 2015). Peer networks and mentor relationships are also essential for the advancement of young scientists (Fox,

\footnotetext{
${ }^{1}$ In this work we use some terms for racial and ethnic minority groups interchangeably: Black and African American; Latinx, Hispanic, and Latin American; and Native American and Indigenous. When referencing other work, we use the term used in that paper.
} 
2001)]; these relationships may arise naturally for certain young men with their senior colleagues but are unavailable to women and minorities.

Implicit bias (both gender and race) impacts people in STEM when seeking mentorship (Milkman et al. 2015), in terms of classroom opportunities (Eddy et al. 2014; 2015; Grunspan et al. 2016), and in workplace conflict and stereotype issues (Williams et al. 2014; 2016; Rios \& Stewart, 2015). Women and scientists of color also experience more workplace incivilities (Cortina et al., 2013; Kabat-Farr \& Cortina, 2012)

Harassment in science is also a problem for retaining women and, especially, women of color. Clancy et al. (2017) surveyed the workplace experiences of Astronomers and Planetary Scientists. They found that $27 \%$ of white women and $40 \%$ of women of color in the sample have felt unsafe at work due to their gender. Further, $28 \%$ of women of color have felt unsafe due to their race. This study demonstrates the value in considering the additional factors that affect the participation of women of color and other groups that are intersections of the demographic questions usually asked.

In addition to cultural barriers, studies of organizations demonstrate that environments with fewer than $30 \%$ minorities are subject to devastating interpersonal dynamics that punish those same minority individuals for their participation. $15 \%$ or fewer minorities invoke a tokenist environment, where individuals are negatively impacted by their heightened visibility (Kanter, 1993).

\subsection{Effect of declining selection rates}

In the last decade, selection rates for NASA scientific research proposals have declined ${ }^{2}$. Planetary science research funding, which is sourced primarily from NASA, is insufficient to support the growing size of the planetary science community (Castillo-Rogez et al., 2020). The effect of declining selection rates is particularly acutely felt by those who are marginalized, without access to a strong network, proposal support, research needs like laboratories and super computers, or name recognition in review panels (Ginther et al., 2011). Moreover, metrics that help boost a proposal's chances, such as previous publications on the topic, are known to be biased. This cycle is self-perpetuating, as lack of grant winning further decreases a scientist's chances of being retained and promoted at their home institution. NASA's goal to support a sustainable and diverse workforce is explicitly stated in multiple high-level NASA strategic documents, such as the NASA Science Vision (Science 2020-2024: A Vision for Scientific Excellence) which identified "Increase the diversity of thought and backgrounds represented across the entire SMD portfolio through a more inclusive environment" as one of its top priorities (Strategy 4.1). It could be argued that diversity, equity, and inclusion are more central to NASA's goals than almost any one particular planetary science investigation. For example, "diversity" is mentioned 7 times in Science Vision and 10 times in the Strategic Plan. In contrast, Europa is mentioned once in the Science Vision and omitted from the Strategic Plan. And yet, declining selection rates are at odds with these goals.

\footnotetext{
${ }^{2}$ See presentations on NASA R\&A made to PAC (such as https://science.nasa.gov/science-pink/s3fs-public/atoms/files/02-Rinehart-PAC-RandA\%200817 20.pdf) and earlier findings presented to the National Academies https://sites.nationalacademies.org/cs/groups/ssbsite/documents/webpage/ssb 175717.pdf
} 


\section{Recommendations}

Rivera-Valentín, et al. (2020) has demonstrated that racial and ethnic minority groups are underrepresented in planetary science at all levels and that the level of underrepresentation generally increases as careers progresses. This shows that there are barriers to entry into every new level of becoming (and remaining) a planetary scientist. Therefore, changes are required at every level, including education and training, recruitment, and promotion and retention. Furthermore, the lack of change in the representation of Latinx and African American scientists demonstrates that current colorblind policies in planetary science are not working and discrimination is still occuring. Instead, we need to implement anti-racist policies to open up participation in planetary science. Additional recommendations can be found in Schmidt et al., 2020.

Recommendation \#1. The decadal survey report should explicitly recognize the statistically significant underrepresentation of people of color-particularly Black / African Americans, Latinx / Hispanics, and American Indian / Indigenous / Alaskan Native people-and that the diversity initiatives of the past several decades have not succeeded in substantially changing this. While diversity efforts aimed at women have increased the representation of women in planetary science, this is not true of African American and Latin American scientists. And yet, many planetary scientists don't realize that these racial and ethinic groups have drastically lower representation than women. Having a finding in the decadal survey report will ensure planetary scientists are more aware of the facts.

Recommendation \#2: NASA should create new programs aimed specifically, and exclusively, to members of underrepresented racial and ethnic minority groups, similar to the programs they currently have for women and girls. The women@NASA website has resources to help women from grade 5 through employment at NASA. A similar page, with similar resources, programs, and funding, should be created for members of underrepresented racial and ethnic minority groups. The programs should include: partnerships with Historically Black Colleges and Universities (HBCUs) and other Minority Serving Institutions (MSIs); support for National organizations for minority scientists, such as National Society for Black Physicists (NSBP) and Society for Advancement of Chicanos/Hispanics and Native Americans in Science (SACNAS); mentorship networks to support racial minorities in STEM, and targeted internships, scholarships, fellowships, summer schools, etc. for scientists of color. Furthermore, NASA should identify ways to fund and recognize mentors for mentoring members of underrepresented groups. Finally, when evaluating the effectiveness of different programs, intersectionality should be considered (e.g., are women of color being included in both programs).

Recommendation \#3.1: NASA should require that groups that receive funding, particularly missions, but also SSERVI (Solar System Exploration Research Virtual Institutes) and other nodes, include specific, meaningful collaborations on the undergraduate/graduate/research level with minority serving institutes, historically black colleges and universities (HBCU), national minority organizations, or Native American tribal council. Because these racial and ethnic groups are so underrepresented, we need to be actively working with members of these groups to encourage more members to pursue planetary science. However, these should not be "outreach" initiatives but "inreaching" initiatives that actually actively bring people into the field as participants from these underrepresented (and underserved) groups. 
Recommendation \#3.2: NASA funding, especially for missions, projects and programs (such as SSERVI), should encourage the development and retention of diverse teams by requiring documentation of and progress on metrics related to diversity, inclusion, and equity. Progress on diversity and inclusion metrics should be monitored in reviews alongside project management and budget reporting. This is detailed further in Norman et al. (2019).

Recommendation \#3.3. NASA should implement a plan for performing climate surveys of large NASA-funded groups, such as mission teams and virtual institutes. Many Universities and corporations have used climate surveys to increase inclusivity in their workplaces. These are generally studies undertaken by professionals to assess the cultural climate in a workplace and how that climate might be improved to be more comfortable for members of underrepresented groups.

Recommendation \#4. NASA should implement a plan for continued, expanded, and funded workforce studies over the next decade. More detailed information and recommendations about this can be found in Rathbun et al. [2020c]. Any policies implemented in order to diversity the planetary workforce, including actions based on the recommendations herein, need to be tracked and compared to metrics and demographics and these results should be published publicly.

Recommendation \#5: NASA should track summary demographics of competed ROSES awards (including R\&A and missions) proposed and awarded and share that information with the planetary community. NASA's NSPIRES grant submission system currently collects demographic data. The fidelity of the data should be improved (by, for example, including genders beyond male and female) and the results need to be shared with the planetary science community. If certain groups receive awards less frequently, then potential inequity should be investigated and changes may need to be made to the review process (for example, dual anonymous review) to address the inequity (Ginther et al., 2011). The demographics should be presented to the community at forums such as the Planetary Science Advisory Committee (PAC), the Committee on Astrobiology and Planetary Science (CAPS), and Agency Night at planetary meetings (LPSC, DPS), etc.

Recommendation \#6.1: Ensure that NASA-sponsored meetings are inclusive by requiring clear policies. These policies should: (1) include a strong anti-harassment statement that includes racial harassment and protects members of underrepresented groups, (2) require that meetings are held in locations that are not dangerous for members of underrepresented groups (including racial and ethnic minority groups, LGBTQ+ scientists, etc.), and (3) require that meetings track and report the demographics of speakers and make changes if certain groups are regularly not being heard.

Recommendation \#6.2: NASA should treat harassment as a form of research misconduct, require mandatory reporting of harassment findings, and follow other recommendations from Zellner et al. (2019). NASA's definition of harassment must include racial harassment.

Recommendation \#7: When discussing diversity, harassment, or related issues, do not focus on only binary gender norms. Several studies on "diversity" focus exclusively on binary gender (see Rathbun et al., 2017 for an example). For a team or group to be truly diverse, it must contain individuals with different identities along multiple axes. Here, we concentrate on race and ethnicity, but see also Vander Kaaden, et al, Strauss et al., and Piatek et al., for other important axes. And it is important to recognize that achieving diversity in one axis does not 
create a general "diversity win" as it does not negate failure to achieve diversity in other axes (e.g., we can celebrate having more women included in a mission team and at the same time complain about and commit to action to redress a lack of underrepresented racial minorities, and visa versa).

Recommendation \#8: Recognize that having multiple axes of diversity can result in an overconstraint on group membership and avoid minimizing demographic/identity-based diversity. When forming a group or team, many different aspects of diversity are deemed important: scientific expertise, discipline, institution-type, career level, etc. Demographics and identity must be treated as equally important and, in some cases, more important. As such, some relaxation of institution and discipline diversity requirements must occur to enable inclusion from groups that are severely underrepresented in the field (such as African American and Latinx planetary scientists). (And please try to avoid trading off one type of demographic/identity diversity for another - there should be room for representation from all axes of minoritized identity.)

Recommendation \#9: Planetary Scientists, their employers, professional societies, and other groups should actively and intentionally find their own ways to assist in improving the diversity in the field. Many blogs, white papers, conference presentations and other publications have listed tools for ways to learn and actions to take (Diniega et al., 2020; Rathbun et al., 2020, etc.). They include paying attention to who is in contact groups, considering diversity when forming teams, and make diversity and inclusion efforts a priority.

\section{References}

AlShebli, B.K., Rahwan, T., \& Woon, W.L. (2018). Nat Comm, 9, 5163.

Bernard, R.E. \& Cooperdock, E.H.G. (2018). Nat Geo, 11, 292-295.

Chen X., \& Weko T. (2009). Report for the US Department of Education, NCES 2009-161.

Clancy, K. B. H., et al. (2017). JGRP, 122, 1610-1623.

Correll, S. J. (2004). Am Soc Rev, 69 (1), 93-113.

Cortina, L.M., et al. (2011). J Management, 39 (6), 1579-1605.

Diniega, S., et al. (2020) Recognizing our colleagues of color in Planetary Science, DPS abstract.

Eddy, S.L., Brownell, S.E., \& Wenderoth, M.P. (2014). CBE Life Sciences Education, 13 (3), 478-92.
Eddy, S.L., et al. (2015). CBE Life Sciences Education, 14 (4).

Ensmenger, N. (2015). Osiris, 30 (1), 38-65.

Foschi, M., et al. (1994). Soc Psych Quart, 57 (4), 326-339.

Fox, M. F. (2001). Gender \& Society, 15 (5), 654-666.

Ginther, D.K., et al. (2011) Science, 333(6045): 1015-1019.

Grunspan, D.Z., et al. (2016). PloS One, 11 (2).

Hendrix, A.R. et al. (2020). LPSC, id.2813. Hofstra, B. et al. (2020). PNAS, 117 (17), 9284-9291.

Kabat-Farr, D., \& Cortina, L.M. (2012). Selective incivility: Gender, race, and the discriminatory workplace. In S. Fox \& T. R. Lituchy (Eds.), New horizons in management. Gender 
and the dysfunctional workplace, $\mathrm{p}$. 120-134. Edward Elgar Publishing.

Kanter, R.M. (1993). Men and women of the corporation. Basic Books.

Milkman, K., Akinola M., \& Chugh D (2015). J Appl Psych, 100 (6), 1678-712.

Neff, G. (2012) Venture labor (MIT Press). Norman, D., et al. (2020). Tying research funding to progress on inclusion. White paper to the Astro2020 Decadal Survey on Astronomy and Astrophysics. BAAS, 51 (7), 14.

O'Brien, L.T., et al. (2015). Cul Div Eth Min Psych, 21 (2), 169-180.

Philips, K.W. (2014). Sci Am, 1 October 2014,

Rathbun, J.A., et al. (2015). DPS, id.312.01.

Rathbun, J.A., et al. (2016). DPS, id.332.01.

Rathbun, J.A., et al. (2018). DPS, id.332.01.

Rathbun, J.A. (2017). Nat Ast, 1, 0148.

Ridgeway, C. L. (2011). Framed by gender: how gender inequality persists in the modern world. Oxford University Press.

Rios, D., \& Stewart, A.J. (2015).J Wom Min Sci Eng, 21 (4), 295-322.

Rudman, L. A., \& Glick, P. (2001). J Soc Iss, 57 (4), 743-762.

Steele, C.M., \& Aronson, J. (1995). J Person Soc Psych, 69(5), 797-811.

Thébaud, S., \& Sharkey A. J. (2016).Soc Sci, $3,1-31$.

Vaughan, D. (2016). The Challenger launch decision: Risky technology, culture, and deviance at NASA. University of Chicago Press.

White, S. et al. (2011). 2011 Survey of the Planetary Science Workforce.
Williams, J. C., K. W. Phillips, and E. V. Hall (2014), Double jeopardy?: Gender bias against women of color in science Rep., UC Hastings College of the Law.

Williams, J. C., K. W. Phillips, and E. V. Hall (2016), J. Res. Gender Stud.,6(1), 11-75.

Zellner, N. et al. (2019). Findings and recommendations from the American Astronomical Society (AAS) Committee on the Status of Women in Astronomy: Towards eliminating harassment in astronomy. White paper to the Astro2020 Decadal Survey on Astronomy and Astrophysics.

\section{Cited White Papers}

Castillo-Rogez, et al. "NASA Research and Analysis..."

Rathbun, J.A., et al. "Enabling the ..."

Rivera-Valentín, E.G., et al. "Who is Missing in Planetary Science?: A demographic study..."

Schmidt et al. "Diversity in action..." 